\title{
Correlative Spectrum Imaging of Organic Photovoltaic Devices using Energy Dispersive X-ray and Electron Energy-Loss Spectroscopy
}

Frank J. Scheltens ${ }^{1}$, Michael F. Durstock ${ }^{2}$, Christopher E. Tabor ${ }^{2}$, Benjamin J. Leever ${ }^{2}$, Michael D. Clark ${ }^{2,3}$, Dennis P. Butcher ${ }^{2,3}$, and David W. McComb ${ }^{1}$

1. Department of Materials Science and Engineering, The Ohio State University, College Road, Columbus $\mathrm{OH} 43210$, USA

2. Materials and Manufacturing Directorate, Air Force Research Laboratory, WPAFB, Ohio 45433, USA

3. UES, Inc., Dayton, Ohio 45432, USA

Due to the possibility of very low cost manufacturing relative to inorganic based devices, bulk heterojunction (BHJ) organic photovoltaic (OPV) based solar cells hold the promise of achieving the highly desirable objective of low cost, clean renewable electrical power via solar energy collection in a flexible device architecture. The cost saving potential of solution processing technologies, such as spin coating and roller casting, with their ability to produce large active areas on flexible substrates at low cost and high throughput $[1,2]$, has driven a very active OPV research and development effort.

Although recent developments in OPV device technology have increased power conversion efficiencies (PCE) into the $7 \%$ to $8 \%$ range $[3,4]$, OPV solar cell technology has not yet achieved the PCE levels of the current inorganic technology. One the most important of the many factors limiting OPV device performance is the morphology of the active layer, which in BHJ OPV devices consists of a bi-continuous network of electron donor and electron acceptor constituents. The domain size, crystallinity and interfacial morphology of this network have a significant impact on exciton disassociation and subsequent electron and hole transport [5] and are, therefore, critical to BHJ OPV device efficiency.

The advent of high speed, high throughput silicon drift detector (SDD) based energy dispersive spectroscopy (EDS) detectors and high speed scanning transmission electron microscopy (STEM) dual electron energy-loss spectroscopy (EELS) have made it possible to rapidly and routinely produce high quality, high resolution spectrum images $[6,7]$. In this presentation we will explore the application of EDS spectrum imaging (EDS SI) and EELS spectrum imaging (EELS SI) to the analysis of BHJ OPV device morphology (Figure 1). Composition and electronic structure variations in the morphology are exploited to elucidate the device structure. [8]

\section{References:}

[1] C.J. Brabec, N.S. Sariciftci, et al., Advanced Functional Materials 11 (2001), 15.

[2] N.S. Lewis, Science 315 (2007), 798.

[3] Y. Liang, Z. Xu, et al., Advanced Materials 22 (2010), E135.

[4] A. Facchetti, Chemistry of Materials 23 (2011), 733.

[5] X. Yang and J. Loos, Macromolecules 40 (2007), 1353. 
[6] D.B. Williams and C.B. Carter in "Transmission Electron Microscopy, Second Edition", (Springer, New York).

[7] R.F. Egerton in "Electron Energy-Loss Spectroscopy in the Electron Microscope, Third Edition", (Springer, New York).

[8] The authors acknowledge the Air Force Office of Scientific Research and the Air Force Research Laboratory Materials and Manufacturing Directorate for funding as well as The Ohio State University Center for Electron Microscopy and Analysis for technical support.

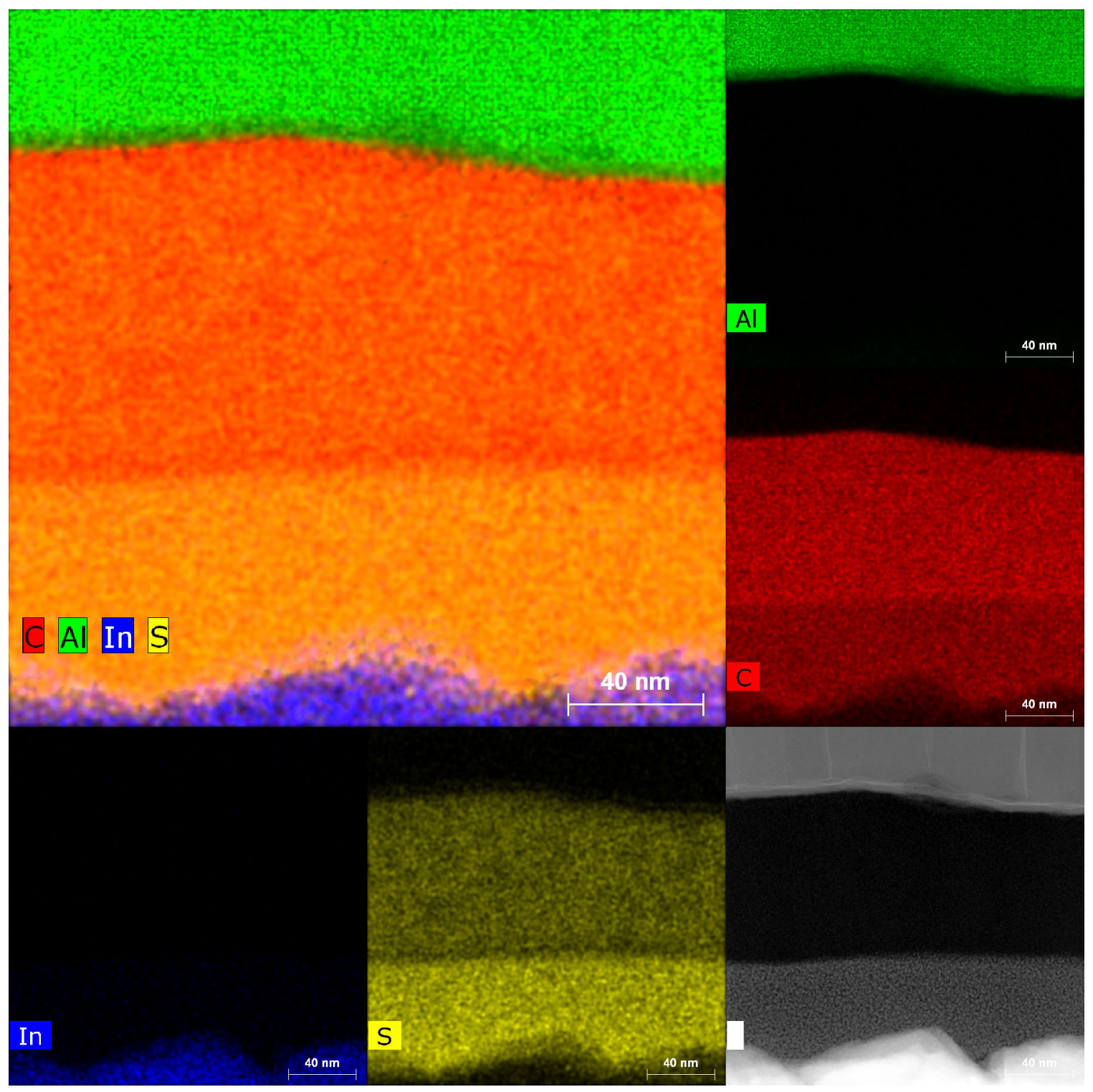

Figure 1. EDS spectrum images of a P3HT:PCBM OPV device cross section showing the composition of the overall $\mathrm{BHJ}$ architecture. 